\title{
A biomechanical comparison between cement packing combined with extra fixation and three- dimensional printed strut-type prosthetic reconstruction for giant cell tumor of bone in distal femur
}

\section{Li Min ( $\nabla$ jacky-min@163.com )}

West China School of Medicine: Sichuan University West China Hospital

Xin $\mathrm{Hu}$

West China School of Medicine: Sichuan University West China Hospital

Minxun Lu

West China School of Medicine: Sichuan University West China Hospital

Yuqi Zhang

West China School of Medicine: Sichuan University West China Hospital

Yitian Wang

West China School of Medicine: Sichuan University West China Hospital

Chongqi Tu

West China School of Medicine: Sichuan University West China Hospital

\section{Research Article}

Keywords: Giant cell tumor, Distal femur, 3D-printed prosthesis, Bone cement, Finite element analysis

Posted Date: January 28th, 2022

DOI: https://doi.org/10.21203/rs.3.rs-1290007/v1

License: (c) (i) This work is licensed under a Creative Commons Attribution 4.0 International License. Read Full License 


\section{Abstract}

Background: The most common reconstruction method for bone defects caused by giant cell tumor of bone (GCTB) is cement packing combined with subchondral bone grafting and extra fixation. However, this method has several limitations involving bone cement and bone graft, which may lead to poor prognosis and joint function. A titanium-based 3D-printed strut-type prosthesis, featured with excellent biocompatibility and osseointegration ability, was developed for this bone defect in our institution. The goal of this study is to comparatively analyze the biomechanical performance of reconstruction methods aimed at the identification of better operative strategy.

Methods: Four different 3D finite element models were created. Model \#1: Normal femur; Model \#2: Femur with tumorous cavity bone defect in the distal femur; Model \#3: Cavity bone defect reconstructed by cement packing combined with subchondral bone grafting and extra fixation; Model \#4: Cavity bone defect reconstructed by 3D-printed strut-type prosthesis combined with subchondral bone grafting. The femoral muscle multiple forces were applied to analyze the mechanical difference among these models by finite element analysis.

Results囚Optimal stress and displacement distribution were observed in the normal femur. Both reconstruction methods could provide good initial stability and mechanical support. Stress distributed unevenly on the femur repaired by cement packing combined with subchondral bone grafting and extra fixation, and obvious stress concentration was found around the articular surface of this femur. However, the femur repaired by 3D-printed strut-type prosthetic reconstruction showed better performance both in displacement and stress distribution, particularly in terms of the protection of articular surface and subchondral bone.

Conclusions: 3D-printed strut-type prosthesis is outstanding in precise shape matching and better osseointegration. Compared to cement packing and extra fixation, it can provide the almost same support and fixation stiffness, but better biomechanical performance and protection of subchondral bone and articular cartilage. Therefore, 3D-printed strut-type prosthetic reconstruction combined with subchondral bone grafting can be an alternative method for the treatment of GCTBs in distal femur.

\section{Introduction}

In 1987, Campanacci et al radiographically classified giant cell tumor of bone (GCTB), a benign but aggressive primary bone tumor, into three grades according to their level of bone destruction $[1,2]$. For grades I or II GCTBs in distal femur, extended intralesional curettage is the standard therapeutic method[1, $3]$, and then the repairment of cavity bone defects seems highly demanded to improve postoperative outcomes.

The most popular reconstruction method is cement packing combined with subchondral bone grafting and extra fixation. Bone cement can perfectly match the osseous voids and provide sufficient mechanical strength, and it has a tumoricidal ability by thermal polymerization, which can adversely damage articular 
cartilage as well $[4,5]$. The subchondral bone grafting $(\geq 1 \mathrm{~cm})$ not only can increase the thickness of subchondral bone if bone union occurs, but also acts as an allograft buffer to prevent thermal damage of the chondrocytes. Although, early complications can be prevented, the long-term effectiveness is unknown. In fact, some patients still developed mechanical failure during long-term follow-up[6]. The main disadvantages include two aspects. Firstly, bone cement is too poorly osteoinductive and osteoconductive to achieve biological reconstruction and osseointegration of graft-cement interface. The different elastic modulus between the bone cement and the graft bone may result in absorbing of the surrounding bone[7]. Secondly, the source of autogenous bone is limited, and those irregular autogenous bone can hardly precisely match the bone defect contour, especially for the patellofemoral and tibiofemoral joints simultaneously.

In our previous study, eight patients with GCTB in the proximal tibia underwent extended curettage and 3D-printed porous implant reconstruction combined with subchondral bone grafting. After a mean followup period of 26 months, osteointegration and satisfactory clinical outcomes were observed in all patients[8, 9]. In the light of experience, a novel 3D-printed strut-type prosthesis has been designed to repair grade I or II GCTBs in distal femur, with great medium-term clinical results. This customized prosthesis has several advantages such as excellent osseointegration ability and low requirement for the amount of bone graft. However, besides excellent osseointegration ability and matchable shape, the mechanical analysis can further verify its effectiveness and safety. Finite element analysis (FEA), a noninvasive method used to investigate the mechanical properties of implant in orthopedical field, has been reported as a promising method to understand the stability and functionality of bone constructs[10,11]. In this experiment, we created FE models and simulated surgical procedures by computer modeling. The aim of our study is to analyze the biomechanical performance of the 3D-printed strut-type prosthesis in comparison with cement packing combined with extra fixation.

\section{Methods}

The CT-scanning data of femur used in this study were derived from a healthy middle-aged male volunteer and a similar-aged patient with GCTB in the distal femur treated with extended curettage followed by cement packing combined with subchondral bone grafting and extra fixation. (Our Ethical Committee authorized the study, and all people provided written informed consent to participate in this investigation).

\subsection{The novel design of 3D-printed strut-type prosthesis}

The 3D-printed strut-type prosthesis was designed by our clinical team, using Solidworks 2016 (Dassault Systèmes SolidWorks Corporation, France). A modular system was applied to minimize the size of cortical windows and to make it convenient for assembling the components in the limited space. The modular system consisted of three components: $₫$ The trapezoid-shaped strut was created to provide effective axial support, and three screws were fixed into the parallel holes through the strut in order to achieve transverse stability. $\otimes$ The turtle shell-shaped strut $A$, and $\otimes$ The turtle shell-shaped strut $B$ were 
designed for maintaining the stability of patellofemoral and tibiofemoral joints and preventing the collapse of the articular surfaces, respectively. A specialized sideway-slider construction has been designed to establish a tight connection between the trapezoid-shaped strut and the turtle shell-shaped strut $B$. The slideway was built on the turtle shell-shaped strut $B$, and the corresponding slider was required to be located on the bottom of the trapezoid-shaped strut. Additionally, this prosthesis was made up of two different materials including the porous titanium (Ti6A14V) and the solid titanium (Ti6A14V). The porous titanium with scaffold structure (dark gray region in Fig. 1) can promote osseointegration of the bone-prosthesis interface while the solid titanium (light gray region in Fig. 1) can provide effective mechanical strength.

\subsection{FE models}

\subsubsection{Model \#1: Normal femur}

The CT-scanning data of the healthy volunteer was reconstructed three-dimensionally as the normal femur model using the software Mimics V17.0 (Materialise Corp. Belgium), as shown in Fig. 2A-D. Then, the initial model was further transformed into a solid model with None-Uniform Ration Basis Spine (NURBS) kyrtograph by the software Geomagic studio 2014 (3D Systems, Inc. USA).

\subsubsection{Model \#2: Femur with tumorous cavity bone defects}

3D CT-scanning data of GCTB patient was used to create tumor model (Fig. 2E-H), then the tumor model and the normal femur model were assembled to simulate the tumorous bone defects in the distal femur with the same size $(59.1 \mathrm{~mm} \times 42.3 \mathrm{~mm} \times 71.2 \mathrm{~mm})$, shape (ellipsoid shape), and location (the distal lateral condyle) as those of the GCTB patient. The femur model with tumorous bone defects was subsequently imported into Solidworks 2016 for the purpose of simulating the surgical procedures, including creating cortical window and intralesional extended curettage. All surgical procedures were performed on the guidance of a senior surgeon (Li Min) from our clinic team (Fig. 3).

\subsubsection{Model \#3: Cavity bone defect reconstructed by cement packing combined with subchondral bone grafting and extra fixation}

Model \#3 was created based on Model \#2, and it required several components, including the distal femur locking plate, the screws, and the bone cement. The distal femur locking plate and the screws were created in Solidworks 2016 on guidance of the manufacturers' specifications. The cavity bone defects after extensive curettage were filled with bone cement, thus the residual cavity in Model \#2 was used to replace the bone cement by Boolean Operation. All components were assembled in Solidworks 2016 (Fig. 4).

\subsubsection{Model \#4: Cavity bone defect reconstructed by 3D- printed strut-type prosthesis combined with subchondral}




\section{bone grafting}

Model \#4 was assembled with the 3D-printed porous strut-type prosthesis and Model \#2 (Fig. 5). The 3Dprinted porous strut-type prosthesis was created by the design data from our previous study: The size of the trapezoid-shaped strut was $12 \mathrm{~mm} \times 28 \mathrm{~mm} \times 38 \mathrm{~mm}$ (max-length $\times$ max-width $\times$ max-height), the size of the turtle shell-shaped strut $A$ was $13 \mathrm{~mm} \times 28 \mathrm{~mm} \times 37 \mathrm{~mm}$ (max-length $\times$ max-width $\times$ max-height), and the size of the turtle shell-shaped strut $B$ was $48 \mathrm{~mm} \times 32 \mathrm{~mm} \times 20 \mathrm{~mm}$ (max-length $\times$ max-width $\times$ max-height). A pore size of $500 \mu \mathrm{m}$ and $70 \%$ porosity was used to simulate the trabecular bone according to data from Torres-Sanchez's study, which confirmed that these parameters could improve osseointegration[12].

\subsection{Material assignment and Mesh}

All materials were set as homogeneous, isotopic, and elastic linearly properties, and the material properties were assigned respectively in Ansys 2019 R3 (ANSYS, Inc. Pennsylvania, USA). The elastic modulus and Poisson's ratio of these materials were listed in Table.1, according to previous study[13]. Furthermore, all materials of FE models were meshed individually, and the element size was $1.0 \mathrm{~mm}$. The number of elements (C3D10) for each model were 2545815 (Model \#1), 501438 (Model \#3), and 1738013 (Model \#4), respectively.

Table.1

Material properties of the bone and implants

\begin{tabular}{|lll|}
\hline Materials & Modulus of Elasticity (MPa) & Poisson's ratio \\
\hline Cortical bone & 13700 & 0.30 \\
\hline Cancellous bone & 1850 & 0.30 \\
Bone cement & 2070 & 0.35 \\
Solid titanium (Ti6Al4V) & 110000 & 0.30 \\
Porous titanium (Ti6Al4V) & 1500 & 0.30 \\
\hline
\end{tabular}

\subsection{Loads and constraints}

A physiological loading representing an instant at forty five percent of the gait cycle (the second part of the single-leg support period) was selected in Ansys 2019 R3. The femoral insertion areas of muscles were mapped on to the surface of each model according to a study by Viceconti et al[14], and the hip joint-femur muscle multiple force was loaded on each model as recommended by Taylor et al[15]. The articular surface of distal condyle was restricted in all directions. (Fig. 6)

In this study, the contact of the cortical bone-trabecular bone interface was assigned as bonded in Ansys 2019 R3. For Model \#3, a frictional coefficient of 0.3 was used for contact boundary with finite sliding between the bone and the distal femur locking plate, as well as the screws. In order to simplify the 
experiment, it's assumed that the bone graft had united with the host bone. However, since the cementgraft bone interface cannot achieve osseointegration, the contacts of this interface was assigned as frictional with the friction coefficient of 0.3 . For Model \#4, the contact of the porous implant-bone interfaces was all set as bonded to simulate the mechanical effects of the osseointegration.

\subsection{Finite element analysis}

All FE models were input to Ansys 2019 R3, and the algorithm was set as Patch Conforming. The results of FEA reveled the biomechanical performance regarding two major features: the displacement and the stress.

\section{Results}

\subsection{The displacement and stress of the normal femur.}

The peak displacement $(12.54 \mathrm{~mm})$ occurred at the center of femoral head and the top of great trochanter, and the displacement gradually decreased from the proximal to distal femur along the femoral shaft (Fig. 7A1). The stress distribution of the normal femur was observed in Fig. 7B. The hip joint force transmitted from the femoral neck to the femoral condyles with stress evenly distributed through the whole femur, and relatively high stress concentration occurred at the lesser trochanter (48.80Mpa) and the anterior distal femur shaft (36.26Mpa). In general, the FE results of the normal femur was in line with those of previous studies[16, 17].

\subsection{The displacement and stress of the femur repaired with cement packing combined with extra fixation}

Compared to the normal femur, similar patterns of displacement were observed in the femur of Model \#3. The displacement value in the lateral femoral condyle was ranged from $0.01-0.06 \mathrm{~mm} \approx 0.01-0.04 \mathrm{~mm}$ (Model \#1), and the peak displacement was $10.43<12.54 \mathrm{~mm}$ (Model \#1), which suggesting that cement packing combined with subchondral bone grafting and extra fixation could achieve stable fixation of the cavity bone defect (Fig. 7A2). However, this reconstruction method changed the transmission mode of the femoral mechanics, and there was a significant difference in stress distribution between Model \#3 and \#1. Stress shielding was located at the distal femoral shaft just beneath the plate, and the Von Mises stress in this region ranged from 1.96 to $8.28 \mathrm{Mpa}$ which was lower than that of the normal femur (6.04$14.56 \mathrm{Mpa})$. Lower Von Mises stress (3.22-6.50Mpa) were also observed in the anterior side of the lateral femoral condyle compared with that of the normal femur (12.54-22.56Mpa). Furthermore, high stress concentration $(84.45 \mathrm{Mpa})$ occurred in the posterior-inferior side of the lateral femoral condyle facing the articular surface of lateral tibiofemoral joint. (Fig. 7C)

\subsection{The displacement and stress of the femur repaired with 3D-printed strut-type prosthesis}


The displacement distribution of the femur repaired by 3D-printed strut-type prosthesis was close to the normal femur (Fig. 7A3), and the displacement value in the lateral femoral condyle varied from 0.01$0.07 \mathrm{~mm} \approx 0.01-0.04 \mathrm{~mm}$ (Model \#1). Like Model \#3, the result suggested that the 3D-printed strut-type prosthesis could provide enough mechanical support for the femur with cavity bone defect. The stress distribution in the femur of Model \#4 was also close to the normal femur. Stress distribution tended to be continuous and homogeneous, and the peak stress located at the site where the trapezoid-shaped strut was connected to the femoral cortex, which was lower than the yield stress of cortical bone. (Fig. 7D)

\subsection{The displacement and stress of the cement-plate-screw fixation system}

In Model \#3, the direction of displacement was vertically downward, with peak displacement concentrated on the top of the implant and decreasing progressively and distally (Fig. 8D). The peak displacement of Model \#3 was $0.81 \mathrm{~mm}$, which was significantly higher than that of Model \#4 $(0.07 \mathrm{~mm})$. The contact stress distributed equally around the locking plate, except the regions where it was contacted with the screws, and the peak value (548.33Mpa) appears at the top screw hole. Interestingly, the peak displacement $(0.57 \mathrm{~mm})$ and the peak stress $(122.16 \mathrm{Mpa})$ of the bone cement located at its posteriorinferior side contacting with the stress concentration area of the femoral condyle mentioned in Fig. 7C.

\subsection{The displacement and stress of the 3D-printed strut-type prosthesis}

For the 3D-printed strut-type prosthesis, the stress distributed mainly on the solid structure located at the bottom of the trapezoid-shaped strut. In contrast, less stress was transmitted to the turtle shell-shaped struts made of porous titanium. Meanwhile, the turtle shell-shaped struts had a smaller displacement compared with the cement-plate-screw system. In addition, the screws fixed at trapezoid-shaped strut can be divided into three regions: the lateral, the middle, and the medial region. Significant displacement and local stress concentration has been found on the middle region of the screws and the the top of the trapezoid-shaped strut (Fig. 8E, F).

\section{Discussion}

\subsection{Traditional reconstruction method faces challenge of protecting articular cartilage and subchondral bone}

Surgical intervention is usually inevitable for the treatment of GCTBs due to their unique biological features, including local aggressiveness, high risk of recurrence, and easily affecting the knee joint of young adults (20-40 years)[4]. Extended intralesional curettage followed by cement packing combined with subchondral bone grafting and plate-screw fixing has been accepted as a main-stream therapy for Campanacci grade I and II GCTBs around the knee. However, several disadvantages of this reconstruction method still exist. Clinically, the exothermic reaction developed during cement hardening can cause 
thermal necrosis of the surrounding graft bone and the articular cartilage[4], which is associated with poor bone graft incorporation. In some cases, bone cement can even accidently leak into the graft bone making the bone healing more difficult. Another drawback for autogenous bone grafting is that the bulk grafts can hardly match the shape of the articular surface, although at the expense of increasing donor site morbidity.

Most importantly, as a non-biological reconstructive material, bone cement failed to grow biologically into the autogenous bone, which cannot achieve effective osteointegration. In our study, this condition was simulated by assigning the contacts of cement-graft bone interface as frictional with the friction coefficient of 0.3 . It should be noted that to simplify the experiment and to reduce the variables, we assumed that the bone graft had united with the host bone right beneath it. However, even under the ideal conditions, high displacement and stress concentration still could be found in the contact area between the bone and the cement, especially in the posterior-inferior side of the lateral femoral condyle facing the articular surface of lateral tibiofemoral joint. High displacement indicated that a poor stability of the surgical reconstruction, and inappropriate stress concentration increased the possibility of pathological fracture and implant reversion[18]. The micromotion and stress concentration may affect the cement mechanical behavior. Consequently, a sclerotic rim occurred, which separate the cement from the surrounding bone[19]. Some researchers believed that this rim decreased the shock-absorbing ability of the subchondral bone, and the articular subchondral bone could be damaged by the fretting wear due to the separation around the cement[20]. The subchondral bone, an effective shock absorber, plays a vital role in maintaining the shape and the stability of the knee joint[21]. Previous studies have stated that a subchondral bone damage could lead to postoperative mechanical failure and worse knee joint function[22, 23]. In a retrospective study by Teng et al.[23], GCTBs patients undergone extensive knee curettage followed by cement packing were classified into different groups according to the extent and depth of the subchondral bone damage, and those from the mild injury group had a lower risk of mechanical failure. In summary, our study confirmed that cement packing combined with subchondral grafting lacks protection of the articular surface and subchondral bone from a mechanical perspective.

\subsection{D-printed strut-type prosthesis improved biomechanical performance and achieved integrated reconstruction}

The FE results of the present study suggested that even though both surgical methods could offer good initial stability and mechanical support for the femur with cavity bone defect after extend curettage, the 3D-printed strut-type prosthesis showed better biomechanical performance both in displacement and stress distribution. Compared to the conventional plate-screw fixation system, the 3D-printed strut-type prosthetic reconstruction provided a near-normal stress distribution in the representative daily activity, the second part of the single-leg support period, in the distal femur after extend curettage. There was no significant displacement found in the bone defect area, and the maximum displacement of the 3D-printed 
porous strut-type prosthesis was $0.07 \mathrm{~mm}$, which was significantly lower than that of the cement-platescrew systems $(0.81 \mathrm{~mm})$. It implied that the 3D-printed porous strut-type prosthesis provided better stability.

Some advantages of the 3D-printed strut-type prosthetic reconstruction, in contrast, may include the following. Firstly, the 3D-printed strut-type prosthesis was customized depending on the results of preoperative imaging, and it could match well with the massive-cavity bone defect as an intrafemur implantation. Thanks to its precise shape matching, less amount of bone graft was required to achieve satisfactory subchondral bone grafting. In comparison, the plate-screw fixation in Model \#3 increased the extra contact area between the femur and the implants, which had changed the way of stress transmission, and the traditional reconstruction method generally suffer from the issue that the source of autogenous bone in bone grafting is limited. Secondly, the 3D-printed strut-type prosthesis with porous scaffold had outstanding advantage of osteointegration capacity. Theoretically, after the 3Dprinted strut type prosthesis implanting into the cavity bone defect, it can form a tight permanent fixation between the bone-prosthesis interface in the long term. The porous scaffold has been proved to be useful in promoting the osteointegration of bone-prosthesis interface[24]. The porous trabecular-like structure with specific size and porosity not only allow ingrowth of bone tissue into the pores, but also provide a similar elastic modulus as the host bone[24,25]. With the development of additive manufacturing technology, the porous scaffold was widely applied in the design and manufacture of titanium-based prosthesis which continues to be used in clinical practice to repair bone defects. Torres-Sanchez's study have identified that a pore size of $500 \mu \mathrm{m}$ and $70 \%$ porosity can be used to simulate the trabecular bone[12]. Therefore, the 3D-printed strut-type prosthesis with porous scaffold was designed with these parameters to improve the osseointegration ability of the implant. Thirdly, the articular cartilages and subchondral bone were effectively protected due to the reasonable design of the modular system: Stress was mainly distributed on the solid structure made of solid titanium and less on the turtle shell-shaped struts made of porous titanium. This favorable biomechanical characteristic was mainly possible thanks to the integrated reconstruction based on the implant's precise shape matching and osseointegration ability. Taken together, our study confirmed that 3D-printed strut-type prosthetic reconstruction combined with subchondral bone grafting could provide enough mechanical support and improve bone ingrowth, which had incomparable advantages in protecting the articular cartilage and subchondral bone over the conventional reconstruction method.

\subsection{The limitation and expectation}

There are some limitations of this study. Firstly, this FE analysis was performed only under single-leg support condition, and analysis under conditions such as walking and stepping up and down stairs will offer more accurate data in future experiments, replicating more realistic events. Secondly, the criterion we used to evaluate the biomechanical performance is the interfragmentary theory, which did not take into account the whole bone healing process, but only the long-term condition. In addition, we noticed a detail that the maximum value of Von mises stress in the normal femur model appears at the edge of 
constricted surface. This finding was unexpected and perhaps can be explained due to fast stress changing at constricted boundaries. For the experiment's precision, they should be ignored when analyzing FE data. Other FE results are consistent with previous studies, which make it a validate control group.

\section{Conclusion}

The 3D-printed strut-type prosthesis can provide effective mechanical support and enhance osseointegration due to its precise shape matching and porous scaffold structure. Additionally, it has incomparable advantages in protecting articular cartilage and subchondral bone compared to traditional reconstruction method. Thus, we recommend 3D-printed strut-type prosthetic reconstruction combined with subchondral bone grafting as a reasonable alternative for treatment of grades I or II GCTBs in distal femur.

\section{Abbreviations}

GCTB: Giant Cell Tumor of Bone; 3D: Three-dimensional; FEA: Finite Element Analysis; NURBS: NoneUniform Ration Basis Spine

\section{Declarations}

\section{Ethics approval and consent to participate}

This study was performed in accordance with the 1964 Helsinki Declaration and was authorized by the Ethics Committee of West China Hospital. All people provided written informed consent to participate in this investigation.

\section{Consent for publication}

Not applicable

\section{Availability of data and materials}

The datasets used and analyzed during the current study are available from the corresponding author on reasonable request.

\section{Competing interests}

The authors declare that they have no competing interests or personal relationships that could have appeared to influence the work reported in this paper. 


\section{Funding}

The institution of one or more of the authors has received, during the study period, funding from Chengdu science and technology project (2017-CY02-00032-GX).

\section{Author's contributions}

$\mathrm{L} M$ and CQ T. designed the experiments; $X \mathrm{H}$ and $M X \mathrm{~L}$ prepared the manuscript; $\mathrm{XH}, \mathrm{YT} \mathrm{W}$, and $\mathrm{YQ} \mathrm{Z}$ performed the experiments; YT W. analyzed the data; CQ T revised the manuscript; and all authors read the manuscript and approved the submission.

\section{Acknowledgments}

Not applicable.

\section{References}

1. Campanacci M, Baldini N, Boriani S, Sudanese A: GIANT-CELL TUMOR OF BONE. Journal of Bone and Joint Surgery-American Volume 1987, 69A(1):106-114. doi:10.2106/00004623-19876901000018 (1987).

2. Sobti A, Agrawal P, Agarwala S, Agarwal M. Giant Cell Tumor of Bone - An Overview. Archives of Bone Joint Surgery-Abjs. 2016;4(1):2-9.

3. Niu XH, Zhang Q, Hao L, Ding Y, Li Y, Xu HR, Liu WF: Giant Cell Tumor of the Extremity Retrospective Analysis of 621 Chinese Patients from One Institution. Journal of Bone and Joint Surgery-American Volume 2012, 94A(5):461-467. doi:10.2106/jbjs.J.01922 (2012).

4. Montgomery C, Couch C, Emory CL, Nicholas R: Giant Cell Tumor of Bone: Review of Current Literature, Evaluation, and Treatment Options. Journal of Knee Surgery 2019, 32(4):331-336. doi:10.1055/s-0038-1675815 (2019).

5. Vaishya R, Pokhrel A, Agarwal AK, Vijay V: Current status of bone cementing and bone grafting for giant cell tumour of bone: a systemic review. Annals of the Royal College of Surgeons of England 2019, 101(2):79-85. doi:10.1308/rcsann.2019.0004 (2019).

6. Benevenia J, Rivero SM, Moore J, Ippolito JA, Siegerman DA, Beebe KS, Patterson FR: Supplemental Bone Grafting in Giant Cell Tumor of the Extremity Reduces Nononcologic Complications. Clinical Orthopaedics and Related Research 2017, 475(3):776-783. doi:10.1007/s11999-016-4755-x (2017).

7. Abdelrahman M, Bassiony AA, Shalaby H, Assal MK: Cryosurgery and impaction subchondral bone graft for the treatment of giant cell tumor around the knee. HSS journal: the musculoskeletal journal of Hospital for Special Surgery 2009, 5(2):123-128. doi:10.1007/s11420-009-9125-8 (2009). 
8. Lu MX, Wang J, Tang F, Min L, Zhou Y, Zhang WL, Tu CQ: A three-dimensional printed porous implant combined with bone grafting following curettage of a subchondral giant cell tumour of the proximal tibia: a case report. Bmc Surgery 2019, 19. doi:10.1186/s12893-019-0491-y (2019).

9. Zhang YQ, Lu MX, Min L, Wang J, Wang YT, Luo Y, Zhou Y, Duan H, Tu CQ: Three-dimensional-printed porous implant combined with autograft reconstruction for giant cell tumor in proximal tibia. Journal of Orthopaedic Surgery and Research 2021, 16(1). doi:10.1186/s13018-021-02446-x (2021).

10. Benli S, Aksoy S, Havitcioglu H, Kucuk M: Evaluation of bone plate with low-stiffness material in terms of stress distribution. Journal of Biomechanics 2008, 41(15):3229-3235. doi:10.1016/j.jbiomech.2008.08.003 (2008).

11. Duda GN, Mandruzzato F, Heller M, Goldhahn J, Moser R, Hehli M, Claes L, Haas NP: Mechanical boundary conditions of fracture healing: borderline indications in the treatment of unreamed tibial nailing. Journal of Biomechanics 2001, 34(5):639-650. doi:10.1016/s0021-9290(00)00237-2 (2001).

12. Torres-Sanchez C, Al Mushref FRA, Norrito M, Yendall K, Liu Y, Conway PP: The effect of pore size and porosity on mechanical properties and biological response of porous titanium scaffolds. Materials Science \& Engineering C-Materials for Biological Applications 2017, 77:219-228. doi:10.1016/j.msec.2017.03.249 (2017).

13. Tang F, Zhou Y, Zhang WL, Min L, Shi R, Luo Y, Duan H, Tu CQ: All-polyethylene tibial components in distal femur limb-salvage surgery: a finite element analysis based on promising clinical outcomes. Journal of Orthopaedic Surgery and Research 2017, 12. doi:10.1186/s13018-017-0555-6 (2017).

14. Viceconti $M$, Ansaloni $M$, Baleani $M$, Toni $A$ : The muscle standardized femur. a step forward in the replication of numerical studies in biomechanics. Proceedings of the Institution of Mechanical Engineers Part H-Journal of Engineering in Medicine 2003, 217(H2):105-110. doi:10.1243/09544110360579312 (2003).

15. Taylor WR, Ploeg H, Hertig D, Warner MD, Clift SE: Bone remodelling of a proximal femur with the thrust plate prosthesis: an in vitro case. Computer methods in biomechanics and biomedical engineering 2004, 7(3):131-137. doi:10.1080/10255840410001712108 (2004).

16. Peng MJQ, Cao XW, Chen HY, Hu Y, Li XX, Lao YQ, Bai B: Intralesional curettage versus prosthetic replacement for bone tumors - a finite element analysis case of limb salvage simulation in biomechanics. Computer Methods and Programs in Biomedicine 2021, 198. doi:10.1016/j.cmpb.2020.105775 (2021).

17. Phillips ATM: The femur as a musculo-skeletal construct: A free boundary condition modelling approach. Medical Engineering \& Physics 2009, 31(6):673-680. doi:10.1016/j.medengphy.2008.12.008 (2009).

18. Peng MJQ, Xu HW, Chen HY, Lin Z, Li XX, Shen CL, Lau YQ, He EX, Guo YM: Biomechanical analysis for five fixation techniques of Pauwels-III fracture by finite element modeling. Computer Methods and Programs in Biomedicine 2020, 193. doi:10.1016/j.cmpb.2020.105491 (2020).

19. Gaston CL, Bhumbra R, Watanuki M, Abudu AT, Carter SR, Jeys LM, Tillman RM, Grimer RJ: Does the addition of cement improve the rate of local recurrence after curettage of giant cell tumours in bone? 
Journal of Bone and Joint Surgery-British Volume 2011, 93B(12):1665-1669. doi:10.1302/0301620x.93b12.27663 (2011).

20. Welch RD, Berry BH, Crawford K, Zhang H, Zobitz M, Bronson D, Krishnan S: Subchondral defects in caprine femora augmented with in situ setting hydroxyapatite cement, polymethylmethacrylate, or autogenous bone graft: biomechanical and histomorphological analysis after two-years. Journal of Orthopaedic Research 2002, 20(3):464-472. doi:10.1016/s0736-0266(01)00124-3 (2002).

21. Yan X, Chen Y-R, Song Y-F, Yang M, Ye J, Zhou G, Yu J-K: Scaffold-Based Gene Therapeutics for Osteochondral Tissue Engineering. Frontiers in Pharmacology 2020, 10. doi:10.3389/fphar.2019.01534 (2020).

22. Ayerza MA, Aponte-Tinao LA, Farfalli GL, Restrepo CAL, Muscolo DL: Joint Preservation after Extensive Curettage of Knee Giant Cell Tumors. Clinical Orthopaedics and Related Research 2009, 467(11):2845-2851. doi:10.1007/s11999-009-0913-8 (2009).

23. Teng WSY, Lin P, Li Y, Yan XB, Li HY, Li BH, Wang Z, Wu Y, Wang SD, Zhou XZ, et al: Bone combined cement grafting in giant cell tumor around the knee reduces mechanical failure. International Orthopaedics 2019, 43(2):475-482. doi:10.1007/s00264-018-3939-2 (2019).

24. Chen YH, Frith JE, Dehghan-Manshadi A, Attar H, Kent D, Soro NDM, Bermingham MJ, Dargusch MS: Mechanical properties and biocompatibility of porous titanium scaffolds for bone tissue engineering. Journal of the Mechanical Behavior of Biomedical Materials 2017, 75:169-174. doi:10.1016/j.jmbbm.2017.07.015 (2017).

25. Ding $X$, Liu X, Chen J, Chen S: Research progress of porous tantalum in bone tissue engineering. Chinese journal of reparative and reconstructive surgery 2018, 32(6):753-757. doi:10.7507/10021892.201711040 (2018).

\section{Figures}

\section{Figure 1}

Diagram of the novel design of 3D-printed strut-type prosthesis: $(A)$ the detached prosthesis, $(B)$ the assembled prosthesis.

\section{Figure 2}

The reconstruction of the normal femur model and the tumor model: (A) Coronal, (B) sagittal, and (C) axial CT images of the normal femur. (D) The normal femur model. (E) Coronal, (F) sagittal, and (G) axial CT images of the femur with GCTB. $(H)$ The tumor model. 


\section{Figure 3}

Diagram of the femur model with tumorous bone defects. The surgical procedures, creating cortical window and intralesional extended curettage, had been simulated by executing modules of Offsetting polygons and Boolean Operation in Solidworks 2016.

\section{Figure 4}

Diagram of the cement packing combined with fixation reconstruction model: (A) Front view, (B) Side view, and (C) Back view

\section{Figure 5}

Diagram of the 3D-printed strut-type prosthetic reconstruction model: (A) Front view, (B) Side view, and (C) Back view.

\section{Figure 6}

The hip joint-femur muscle multiple force was applied to these femur models, and the distal condyle articular surface was fixed.

\section{Figure 7}

The displacement and stress distribution of femurs: (A1-3) The displacement of femur in Model \#1, \#3, and \#4. (B) The stress distribution of the normal femur. (C) The stress distribution of femur in Model \#3, stress shielding (yellow box) and stress concentration (red box) occurred. (D) The stress distribution of femur in Model \#4.

\section{Figure 8}

The displacement and stress distribution of implants: (A, B) The stress and displacement distribution of the bone cement, high stress concentration and displacement occurred at the bottom of the cement near 
the articular surface. (C, D) The stress and displacement distribution of the locking plate and screws. (E, F) The stress and displacement distribution of the 3D-printed strut-type prosthesis. 